\title{
Effect of Biosynthesized Silver Nanoparticles on Staphylococcus aureus Biofilm Quenching and Prevention of Biofilm Formation
}

\author{
Pratik R. Chaudhari*, Shalaka A. Masurkar, Vrishali B. Shidore, Suresh P. Kamble
}

(Received 20 November 2011; accepted 02 March 2012; published online 19 March 2012).

\begin{abstract}
The development of green experimental processes for the synthesis of nanoparticles is a need in the field of nanotechnology. The synthesis of silver nanoparticles was achieved using Bacillus cereus supernatant and $1 \mathrm{mM}$ silver nitrate. $100 \mathrm{mM}$ glucose was found to quicken the rate of reaction of silver nanoparticles synthesis. UV-visible spectrophotometric analysis was carried out to assess the synthesis of silver nanoparticles. The synthesized silver nanoparticles were further characterized by using Nanoparticle Tracking Analyzer (NTA), Transmission Electron Microscope and Energy Dispersive X-ray spectra. These silver nanoparticles showed enhanced quorum quenching activity against Staphylococcus aureus biofilm and prevention of biofilm formation which can be seen under inverted microscope $(40 \mathrm{X})$. The synergistic effect of silver nanoparticles along with antibiotics in biofilm quenching was found to be effective. In the near future, silver nanoparticles could be used in the treatment of infections caused by highly antibiotic resistant biofilm.
\end{abstract}

Keywords: Silver Nanoparticles; Green Synthesis; Bacillus cereus; Biofilm; Quorum Quenching

Citation: Pratik R. Chaudhari, Shalaka A. Masurkar, Vrishali B. Shidore and Suresh P. Kamble, "Effect of Biosynthesized Silver Nanoparticles on Staphylococcus aureus Biofilm Quenching and Prevention of Biofilm Formation", Nano-Micro Lett. 4 (1), 34-39 (2012). http://dx.doi.org/10.3786/nml.v4i1.p34-39

\section{Introduction}

The field of nanotechnology is one of the most active areas of research in modern material science. Nanoparticles are being considered to be the fundamental building blocks of nanotechnology. Nanotechnology is interdisciplinary which includes physics, chemistry, biology, material science and medicine. Instead of using toxic chemicals for the reduction and stabilisation of metallic nanoparticles, the use of various biological entities has received considerable attention in the field of nanobiotechnology [1]. Biological methods are regarded as safe, cost-effective, sustainable and environment friendly processes for the synthesis of nanoparticles [2]. Silver nanoparticles have been successfully synthesized using various bacteria [3-5], fungi $[6,7]$ and plants $[8,9]$.

The term biofilm has been introduced to designate the thin layered condensations of microbes (e.g. bacteria, fungi, protozoa) that may occur on various surface structures in nature. Free-floating bacteria existing in an aqueous environment, so-called planktonic microorganisms are a prerequisite for biofilm formation. Such films may thus become established on any organic or inorganic surface substrate where planktonic microorganisms prevail in a water-based solution. In dental contexts, a well-known and extensively studied biofilm structure is established during the attachment of bacteria to teeth to form dental plaque. Here, bacteria free in saliva (planktonic organisms) serve as the primary source for the organization of this specific biofilm [10]. The excretion of adhesive substances viz. polysaccha-

Center for Biotechnology, Pravara Institute of Medical Sciences, Loni-413736, Ahmednagar (MS), India.

*Corresponding author. E-mail: pratikchaudhari11@gmail.com 
rides and proteins is crucial for the initial attachment of organisms as well as for holding the biofilm bacteria together. The structure per se will then provide protection and may allow a better resistance to adverse external influences for the organisms incorporated as compared with the planktonic state [11]. Phenotypically the organisms may also take on a different character. In addition, a growing body of knowledge suggests that organisms in biofilms assume a stronger pathogenic potential than those in a planktonic state. From these aspects, the formation of biofilm carries particular clinical significance because not only host defense mechanisms, but also therapeutic efforts including chemical and mechanical anti-microbial treatment measures, have a most difficult task to deal with organisms that are gathered in a biofilm [12].

In present study, silver nanoparticles are synthesized using $B$. cereus supernatant. The study deals with the effect of biologically synthesized silver nanoparticles on $S$. aureus biofilm quenching and prevention of $S$. aureus biofilm formation. Diseases such as endocarditis, osteomyelitis and medical-device related infections are caused by $S$. aureus biofilms and are not readily treatable with antibiotics. In fact, biofilms are resistant to antibiotic levels 10- to 1,000-fold higher than planktonic, or free-floating, bacteria [13]. Thus, researchers are focusing on silver nanoparticles for the treatment of infections caused by biofilms.

\section{Materials and methods}

\subsection{Collection of B. cereus Supernatant}

Bacillus cereus (ATCC 8640) was obtained from Department of Botany, Government Institute of Science, Aurangabad (MS), India. Bacillus cereus was grown on sterile Luria Bertani (LB-Casein enzymatic hydrolysate $10 \mathrm{gm} / \mathrm{l}$, Yeast extract $5 \mathrm{gm} / \mathrm{l}$, Sodium chloride 10 $\mathrm{gm} / \mathrm{l}$, Agar $25 \mathrm{gm} / \mathrm{l}, \mathrm{pH} 7.5 \pm 0.2$ ) agar plate $\left(\right.$ at $37^{\circ} \mathrm{C}$ ). The single isolated colony was inoculated in sterile LB broth and incubated at $37^{\circ} \mathrm{C}$ at $200 \mathrm{rpm}$ in rotary shaker for 24 hours. After incubation, culture was centrifuged at $4500 \mathrm{rpm}$ for 10 minutes, the supernatant was collected.

\subsection{Synthesis of silver nanoparticles using cul- ture supernatant of B.cereus}

Bacterial supernatant was mixed with $1 \mathrm{mM}$ silver nitrate in $1: 1$ proportion. The $\mathrm{pH}$ of solution was adjusted to 9.0-9.5 [14] and then resultant solutions were kept in rotary shaker $(200 \mathrm{rpm})$ at $37^{\circ} \mathrm{C}$ till the change in the colour of the solution was observed.
2.3 Synthesis of silver nanoparticles using culture supernatant of $B$. cereus along with 100 $\mathrm{mM}$ glucose

Bacterial supernatant :1mM silver nitrate $: 100 \mathrm{mM}$ glucose were mixed in 1:1:1 proportion. The $\mathrm{pH}$ of solution was adjusted to 9.0-9.5. The resultant solution was kept in rotary shaker $(200 \mathrm{rpm})$ at $37^{\circ} \mathrm{C}$.

\subsection{UV-visible spectrophotometer analysis}

After observing colour change, the sample was subjected to mild sonication for 10 minutes. The bioreduction of silver ions in aqueous solution was monitored by UV-Vis spectra of the solution between $300 \mathrm{~nm}-600 \mathrm{~nm}$ using Thermo- Biomate 3 UV-visible spectrophotometer. Distilled water was taken to adjust the baseline.

\subsection{Nanoparticle Tracking Analyzer (NTA) Measurements}

NTA analysis was carried out by using NanosightLM20 instrument. $0.3 \mathrm{ml}$ samples were introduced to the viewing unit using a disposable syringe and enhanced by a near perfect black background; particles appear individually as point-scatterers moving under Brownian motion.

\subsection{Transmission Electron Microscopy (TEM) and Energy Dispersive X-ray Spectra (EDX) analysis}

TEM analysis of the sample was done using PHILIPS- CM 200 instrument operated at an accelerating voltage of $200 \mathrm{kV}$ with resolution of $0.23 \mathrm{~nm}$. A drop of solution was placed on carbon coated copper grid and later exposed to infrared light (45 minutes) for solvent evaporation. The EDX analysis was carried out using JEOL JSM 7600F.

\subsection{In vitro synthesis of Staphylococcus aureus biofilm}

The biofilm related studies have been performed with the modification of protocol suggested by KaplanRagunath-Ramasubbu-Fine [15] and King-Tatum [16]. Tryptone soy broth (TSB- Pancreatic digest of casein 17 $\mathrm{gm} / \mathrm{l}$, Papaic digest of Soyabean meal $3 \mathrm{gm} / \mathrm{l}$, Sodium chloride $5 \mathrm{gm} / \mathrm{l}$, Dipotassium hydrogen phosphate 2.5 $\mathrm{gm} / \mathrm{l}$, Dextrose $2.5 \mathrm{gm} / \mathrm{l}, \mathrm{pH} 7.3 \pm 2$ ) with $1 \%$ of glucose was inoculated with single isolated colony of $S$.aureus (NCIM 5022) and was incubated at $37^{\circ} \mathrm{C}$ for $24 \mathrm{hrs}$. After incubation culture was diluted with fresh TSB$1 \%$ glucose in 5:100 proportion, then $200 \mu \mathrm{l}$ of diluted culture was added in 96 well micro titer plate and in- 
cubated at $37^{\circ} \mathrm{C}$ for 48 hrs $[15,16]$.

\subsection{Addition of quenching agent in Staphylococ- cus aureus biofilm}

First column of wells was served as positive control in which $25 \mu$ l of $20 \%$ sodium dodecyl sulphate was added, second column of wells was served as negative control (untreated biofilm). In the third column of wells, $50 \mu \mathrm{l}$ of concentrated silver nanoparticles were added. About $50 \mu \mathrm{l}$ of different antibiotics solution, Gentamicin $(10 \mu \mathrm{g} / \mathrm{ml})$ and Chloramphenicol $(20 \mu \mathrm{g} / \mathrm{ml})$ were added in fourth and fifth column of wells respectively. To check synergistic effect of silver nanoparticles along with antibiotics $25 \mu \mathrm{l}$ of silver nanoparticles along with $25 \mu \mathrm{l}$ of above antibiotics were added in sixth and seventh column of wells separately. In eighth column of wells $50 \mu \mathrm{l}$ of $1 \mathrm{mM} \mathrm{AgNO}_{3}$ was added. The plate was incubated at $37^{\circ} \mathrm{C}$ for overnight.

After incubation, plate was washed with $200 \mu \mathrm{l}$ of phosphate buffer saline ( $\mathrm{pH}-7.2)$ to remove floating bacteria. Micro titer plate was stained with $100 \mu \mathrm{l}$ of 0.1 $\%$ crystal violet for 2 minutes then washed with distilled water. Then add $200 \mu \mathrm{l}$ of $33 \%$ acetic acid and incubate for 5 minutes. Then Plate was properly dried in laminar air flow cabinet. Plate was analysed under inverted microscope $(40 \mathrm{X})$ to record the results. The whole experiment has been performed in triplicate to ensure the reproducibility of the results.

\subsection{Effect of silver nanoparticles in prevention of Staphylococcus aureus biofilm formation}

Overnight grown Staphylococcus aureus culture was diluted 1:100 in fresh Tryptone soya broth and allowed to grow for 1 hour. About $200 \mu$ l of diluted culture was added to micro titer plate. One column of wells was served as positive control ( $25 \mu \mathrm{l}$ of $20 \%$ sodium dodecyl sulphate); second column of wells was served as negative control (untreated culture). In the third column of wells, $50 \mu \mathrm{l}$ of concentrated silver nanoparticles were added. In fourth column of well $50 \mu \mathrm{l}$ of $1 \mathrm{mM} \mathrm{AgNO}_{3}$ was added. Plate was incubated at $37^{\circ} \mathrm{C}$ for 3 days [17]. Washing and staining was done by method mentioned above.

\section{Results and discussion}

\subsection{Synthesis of silver nanoparticles using $B$. cereus culture supernatant}

Colour change was observed upon mixing the $B$. cereus culture supernatant with aqueous solution of 1 $\mathrm{mM}$ silver nitrate in 1:1 (pH 9.0-9.5), which was incubated at $37^{\circ} \mathrm{C}$ for 24 hours (Fig. 1). Flask containing culture supernatant of $B$. cereus along with $100 \mathrm{mM}$ glucose and silver nitrate showed intense colour change than the method of synthesizing silver nanoparticles using culture supernatant alone. Intense colour change suggested that the synthesis of silver nanoparticles may be more in the case of this method.

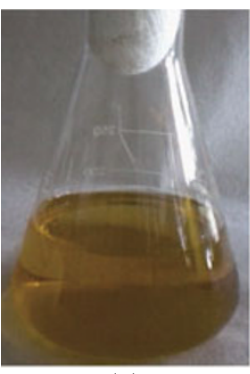

(a)

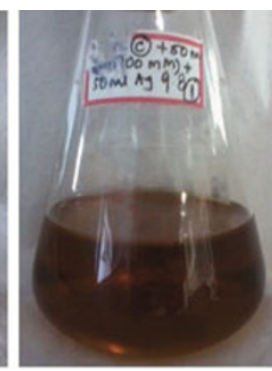

(b)

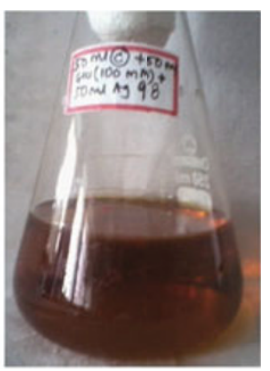

(c)
Fig. 1 (a) Initial reaction mixture containing B. cereus supernatant and $1 \mathrm{mM}$ silver nitrate in 1:1 ratio; (b) Color change of reaction mixture containing $B$. cereus supernatant and $1 \mathrm{mM}$ silver nitrate in 1:1 ratio; (c) Color change of reaction mixture containing $B$. cereus supernatant, $1 \mathrm{mM}$ silver nitrate and $100 \mathrm{mM}$ glucose in 1:1:1 ratio.

\subsection{UV-visible spectrophotometer analysis}

The synthesis of silver nanoparticles by reduction of aqueous metal ions during exposure of $B$. cereus supernatant can be easily monitored by using UV-visible spectrophotometer. Figure 2 illustrates the absorbance spectra of reaction mixture containing aqueous solution of $1 \mathrm{mM}$ silver nitrate and $B$. cereus culture supernatant after incubation. Reaction mixture showed an absorbance peak at around $425 \mathrm{~nm}$, which is characteristic of silver nanoparticles, due to its surface plasmon resonance absorption band [18]. In case of the synthesis mediated by $B$. cereus supernatant in the presence of $100 \mathrm{mM}$ glucose, the absorbance peak was obtained at around $425 \mathrm{~nm}$.

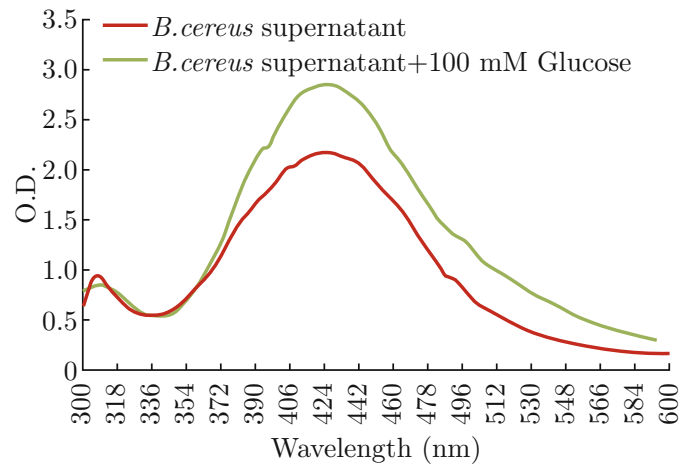

Fig. 2 UV-visible spectrophotometer analysis of silver nanoparticles synthesized using $B$. cereus supernatant and $B$. cereus supernatant in presence of $100 \mathrm{mM}$ glucose.

\subsection{NTA measurements}

NTA measurements revealed that the mean size of synthesized silver nanoparticles was found to be $39 \mathrm{~nm}$ with concentration of $7.8 \times 10^{10}$ particles $/ \mathrm{ml}$ in case of 


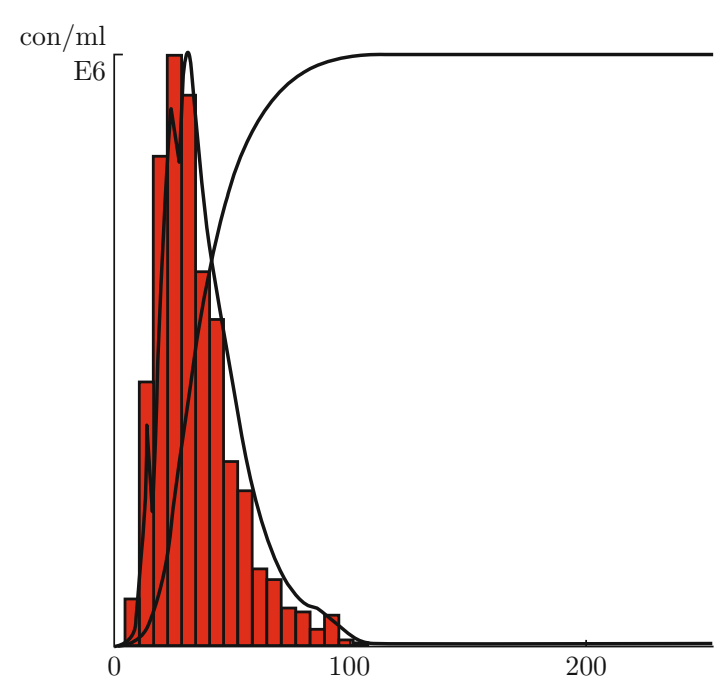

Fig. 3 Frequency size distribution graph of silver nanoparticles synthesized using $B$. cereus supernatant. $\mathrm{X}$ axis: particle size in $\mathrm{nm}$; Y axis: concentration $/ \mathrm{ml} \times 10^{6}$.
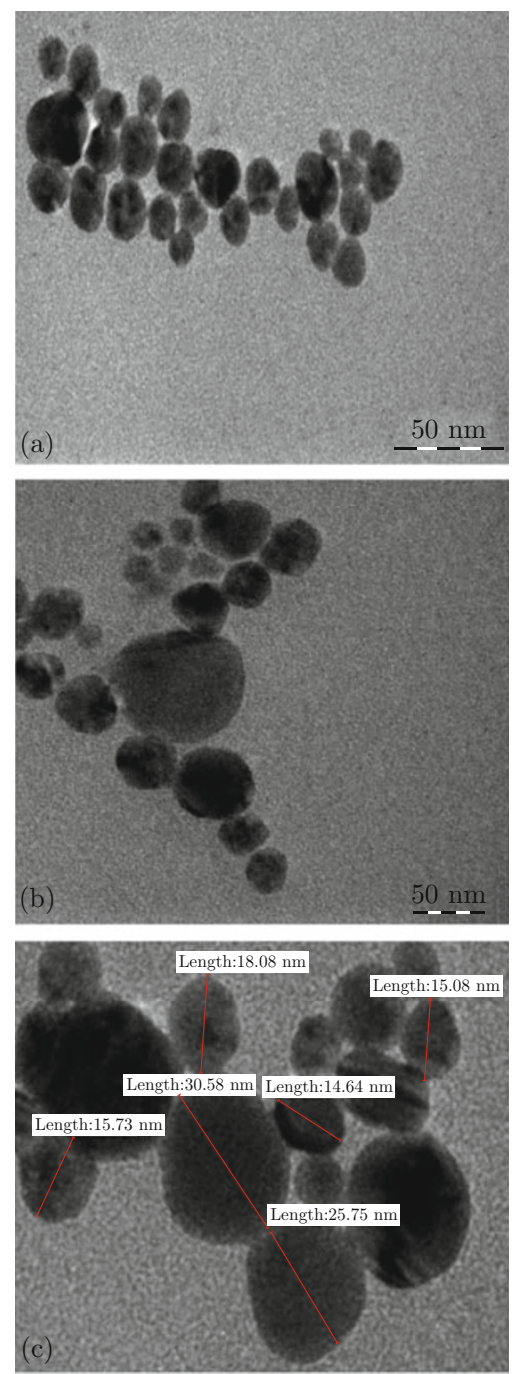

Fig. 4 TEM micrograph of silver nanoparticles synthesized using (a) B. cereus supernatant; (b) and (c) B. cereus supernatant and $100 \mathrm{mM}$ glucose.
Bacterial supernatant mediated synthesis. The mean size of silver nanoparticles synthesized using bacterial supernatant in presence of glucose was found to be $32 \mathrm{~nm}$ with concentration of $10.4 \times 10^{10}$ particles $/ \mathrm{ml}$ (Fig. 3).

\subsection{TEM and EDX analysis}

TEM analysis revealed that the silver nanoparticles are prominently spherical (Fig. 4). The silver nanoparticles were found to be well dispersed from each other. The EDX analysis revealed that the silver is present in the solution (Fig. 5). The silver content in the particles was found to be $70.29 \%$.

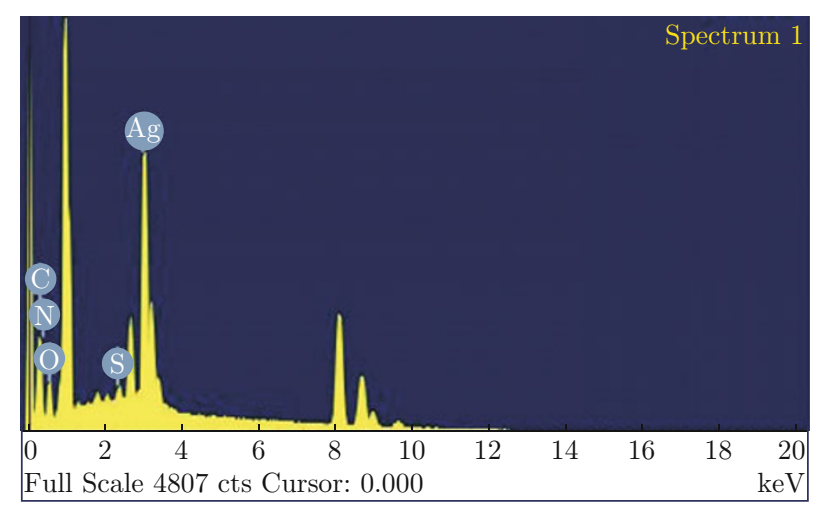

Fig. 5 EDX spectra of silver nanoparticles solution.

\subsection{Quorum quenching Effect of silver nanoparticles on Staphylococcus aureus Biofilm}

Silver nanoparticles showed quenching of biofilm which can be compared with negative control antibiotics failed to show biofilm quenching alone which can be compared with positive control. But synergistic effect of silver nanoparticles and antibiotic showed the biofilm quenching. $1 \mathrm{mM}$ silver nitrate also did not show any quenching of biofilm. (Fig. 6(a-h))

\subsection{Effect of silver nanoparticles in Prevention of Staphylococcus aureus biofilm Formation}

Silver nanoparticles showed prevention of biofilm formation which can be compared with negative control. Where as positive control showed distinct biofilm formed which indicates that silver nanoparticles prevent formation of bacterial biofilm. (Fig. 6(i)) It is known that the excretion of adhesive substances viz. polysaccharides and proteins is crucial for the initial attachment of organisms as well as for holding the biofilm bacteria together [11]. The silver nanoparticles might be involved in neutralizing these adhesive substances, thus preventing biofilm formation. 

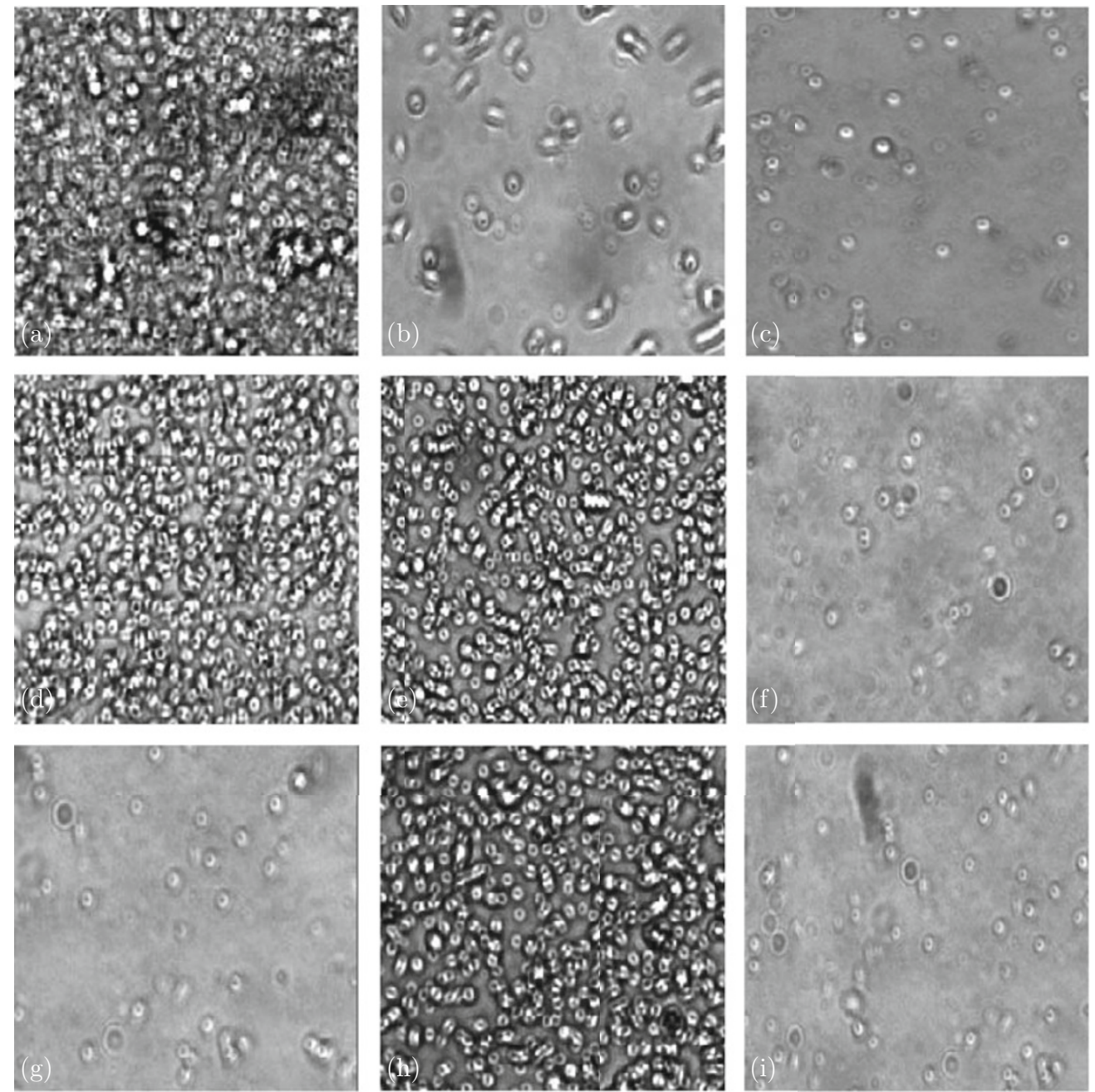

Fig. 6 Effect of silver nanoparticles in biofilm quenching and prevention of $S$. aureus biofilm formation under inverted microscope (40X) (a): Negative control (intact biofilm); (b): Positive control (Biofilm Quenched using 20\% SDS); (c): Silver nanoparticles; (d): Gentamicin $(10 \mu \mathrm{g} / \mathrm{ml})$; (e): Chloramphenicol $(20 \mu \mathrm{g} / \mathrm{ml})$; (f): Silver nanoparticles + Gentamicin $(10 \mu \mathrm{g} / \mathrm{ml}) ;(\mathrm{g})$ : Silver nanoparticles+ Chloramphenicol $(20 \mu \mathrm{g} / \mathrm{ml}) ;(\mathrm{h}): 1 \mathrm{mM}$ silver nitrate; (i): Silver nanoparticles in prevention of biofilm formation.

\section{Conclusion}

In the present study, silver nanoparticles have been synthesized using $B$. cereus supernatant alone and $B$. cereus supernatant in the presence of glucose. The increased synthesis of silver nanoparticles was achieved in case of $B$. cereus supernatant in the presence of glucose. The exact role played by glucose in enhancing the rate of synthetic reaction of silver nanoparticles will be the field of research interest.

In the present study, effect of silver nanoparticles in biofilm quenching and prevention of biofilm formation has been demonstrated. From the results it can be concluded that silver nanoparticles can be involved in biofilm quenching or prevention of biofilm formation. However, antibiotics failed to show any quenching of bacterial biofilm. Synergistic effect of silver nanoparti- cles along with antibiotics in biofilm quenching has also been demonstrated in this study. The exact mechanism of action of silver nanoparticles in biofilm related studies is yet to be demonstrated. In the near future, silver nanoparticles may play a major role in the treatment of infections caused by highly antibiotic resistant biofilm.

\section{Acknowledgments}

We are thankful to Dr. Aurobindo Roy, Dr. Suresh Jangle, Dr. Razia Kutty, Mr. Keshav Shinde, Mr. Santosh Suradkar and Mr. Rajesh Raut for their priceless help. We sincerely acknowledge the facilities provided by Institute of Science, Mumbai and SAIF, IIT Mumbai. We are thankful to Pravara Institute of Medical Sciences, Loni (MS), India for the financial support. 


\section{References}

[1] Y. Park, Y. N. Hong, A. Weyers, Y. S. Kim and R. J. Linhardt, IET Nanobiotechnology 5, 69 (2011).

[2] G. Schmid, Chem. Rev. 92, 1709 (1992). http://dx. doi.org/10.1021/cr00016a002

[3] K. Kalimuthu, R. S. Babu, D. Venkataraman, B. Mohd and S. Gurunathan, Colloids Surf. B 65, 150 (2008). http://dx.doi.org/10.1016/j. colsurfb. 2008.02.018

[4] P. R. Chaudhari, S. A. Masurkar, V. B.Shidore and S. P. Kamble, Int. J. Pharma and Bio Sciences 3, 222 (2012).

[5] N. Saifuddin, C. W. Wong and A. N. Yasumira, E-J. Chem 6, 61 (2009).

[6] A. Ahmad, P. Mukherjee, S. Senapati, D. Mandal, M. I. Khan and R. Kumar, Colloids Surf. B 28, 313 (2003). http://dx.doi.org/10.1016/ S0927-7765(02)00174-1

[7] K. Kathiresan., S. Manivannan, M. A. Nabeel and B. Dhivya, Colloids Surf. B 71, 133 (2009). http://dx. doi.org/10.1016/j.colsurfb. 2009.01.016

[8] R. Raut, J. R. Lakkakula, N. Kolekar, V. D. Mendhulkar and S. B. Kashid, Nano-Micro Lett. 2, 106 (2010). http://dx.doi.org/10.5101/nml.v2i2. p106-113

[9] S. A. Masurkar, P. R. Chaudhari, V. B. Shidore and S. P. Kamble, Nano-Micro Lett. 3, 189 (2011). http:// dx.doi.org/10.5101/nml.v3i3.p189-194
[10] G. H. Bowden. and I. R. Hamilton, Crit. Rev. Oral. Biol. Med 9, 54 (1998). http://dx.doi.org/10.1177/ 10454411980090010401

[11] K. Lewis, Antimicrob. Agents Chemother. 45, 999 (2001). http://dx.doi.org/10.1128/AAC.45.4. 999-1007. 2001

[12] J. W. Costerton, P. Stewart and E. P. Greenberg, Science 284, 1318 (1999). http://dx.doi.org/10.1126/ science. 284.5418.1318

[13] D. Monroe, PLoS Biology. 5, 2458 (2007). http://dx. doi.org/10.1371/journal.pbio. 0050307

[14] M. Darroudi, M. B. Ahmad, A. H. Abdlahul, N. A. Ibrahim and K. Shameli, Int. J. Mol. Sci. 11, 3898 (2010). http://dx.doi.org/10.3390/ijms11103898

[15] J. B. Kaplan, C. Ragunath, N. Ramasubbu and H. Daniel, J. Bacteriol. 185, 4693 (2003). http://dx.doi. org/10.1128/JB.185.16.4693-4698. 2003

[16] E. O. King and H. W. Tatum, J. Infect. Dis. 111, 85 (1962). http://dx.doi.org/10.1093/infdis/111. 2.85

[17] K. Raja, N. Selvaraj, E. Krishnamoorthi and B. K. Singh, IJPI's J. Biotechnology Biotherapeutics. 1, 1 (2011).

[18] S. Akanna, K. V. Prasad, E. K. Elumalai and N. Savithramma, Digest J. Nanomater. Biostruct. 5, 369 (2010). 